\title{
Thin Film and Multilayer Scintillators for Low Voltage Backscattered Electron Imaging in the Scanning Electron Microscope
}

\author{
NC Barbi ${ }^{1}$, MB Tzolov ${ }^{2}$, OE Healy ${ }^{1}$, RB Mott $^{1}$, WM McDonald ${ }^{2}$ \\ ${ }^{1}$ PulseTor LLC, Pennington, NJ USA \\ ${ }^{2}$ Lock Haven University, Department of Geology and Physics, Lock Haven, PA USA
}

Scintillators produce a number of photons proportional to the average energy of the incident electrons. Low energy electron detection using scintillators therefore starts at a disadvantage compared to imaging at higher energies. The predominant scintillator used in electron microscopy is crystalline Ce-doped Yttrium Aluminum Garnet (YAG), a poor electrical conductor that must be coated with a conductive layer on the impact surface to drain the excess charge induced by the very electrons which it must detect. Fortunately, this conductive layer (Al or ITO) can be very thin, on the order of 10-20 $\mathrm{nm}$, and therefore has little effect on electrons with energy greater than $10 \mathrm{kV}$. At low energies, however, absorption in the conductive film becomes a factor. The combined result of lower yield at low energies and the absorption by the conductive layer is that BSE imaging is seldom used below $5 \mathrm{kV}$.

New detector designs, using scintillators coupled to Silicon PhotoMultipliers (SiPMs), allow different sensor segments to be placed around a central hole in the detector, meaning that different scintillator types can be used in the same device [1]. In such "Scintillator on Multiplier" (SoM) detectors, electrically conductive $\mathrm{ZnO}$, for example could be used without coating as a scintillator for low energy electrons in one or more sensor locations, and YAG $(\mathrm{Ce})$ in the remainder. Figure 1 repeats the illustration of a single detector using different scintillator materials. Because there is an SiPM coupled to each scintillator segment, the imaging signal can be arbitrarily derived from a single sensor, one type of sensor, or all the sensors combined.

We have extended this idea further by developing thin films of zinc tungstate (ZT) showing photoluminescence quantum efficiency in the range of 70\%, and which exhibit sufficient conductivity to drain the charge typically encountered in SEM imaging. The nanostructure of these films leads to improved scintillation properties compared to the bulk material, and indeed, compared to $\mathrm{ZnO}$. The absence of an additional top coating allows electrons to directly interact with the surface of the film and produce scintillation. Figure 2 shows improved imaging realized with $\mathrm{ZT}$ at $2 \mathrm{kV}$ compared to YAG, both images being collected simultaneously. The SNR as a function of incident beam energy (Figure 3) shows the advantage of thin ZT for low energy, while confirming the superiority of bulk YAG for higher energies. Figure 4 shows an image taken at $1 \mathrm{kV}$ with ZT. For comparison, the emission spectrum of zinc tungstate is compared with that of YAG and YAP (Yttrium Aluminum Perovskite) in Figure 5.

The improvement provided by thin film ZT for low energies, the superiority of YAG (Ce) for higher energies, and the flexibility of the deposition process together suggest that integrating ZT with YAG into a multilayer structure would be advantageous. We have constructed the first multilayer ZT/YAG scintillator. This structure will combine the efficient interaction of low energy electrons with a surface ZT film and the efficient collection of high energy electrons by the significantly thicker YAG scintillator underneath. This compact scintillator structure can extend the application of BSE imaging 
to low voltage, while maximizing efficiency through optimum use of the available sensor space in the detector.

[1] N.C. Barbi, et al Microsc. and Microanal., 2011
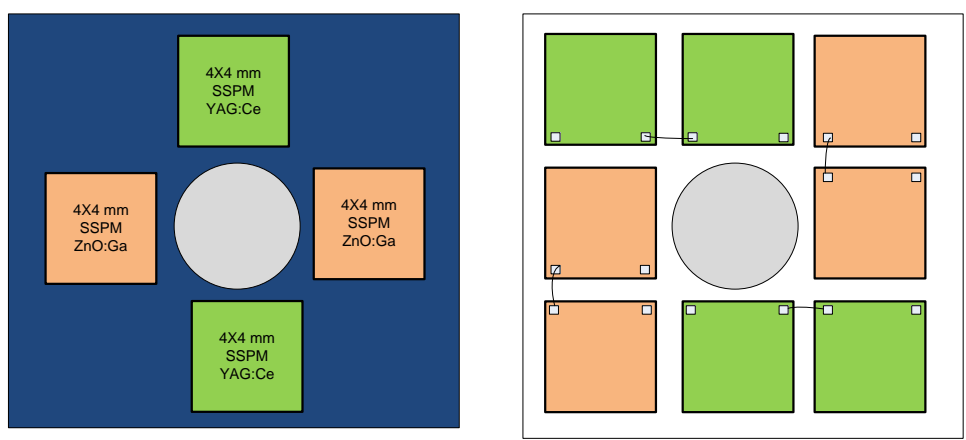

Figure 1: Example of SoM detector configuration having a segmented configuration enabling use of different scintillator materials

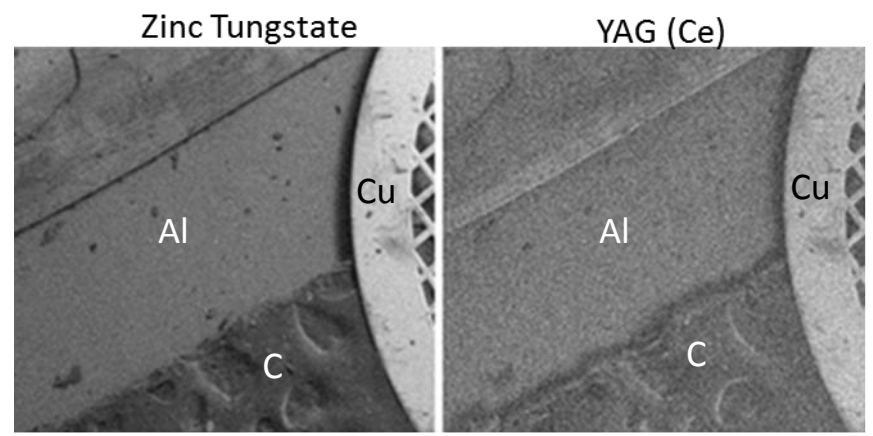

Figure 2: Cu grid - Al stub - C tape $2 \mathrm{kV} 2.5 \mathrm{nA} 80$ second integration time

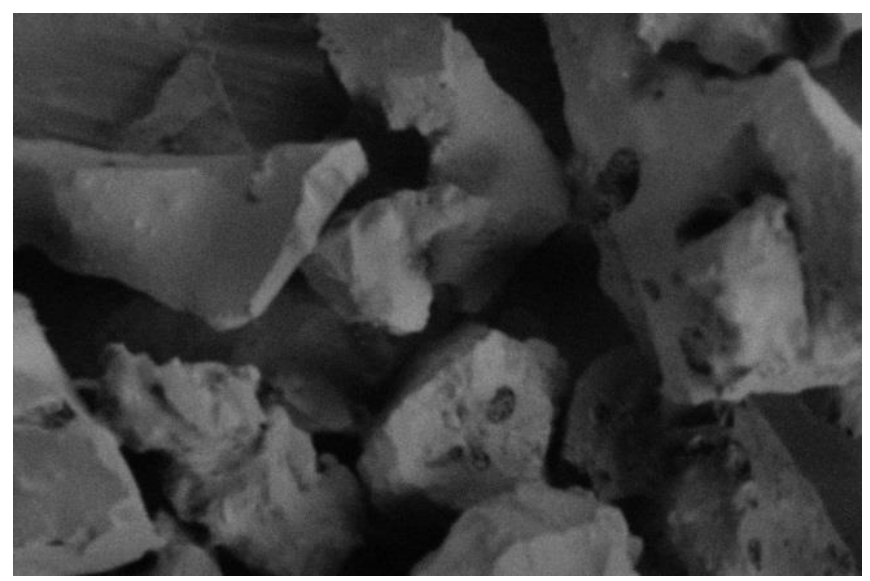

Figure 4: $1 \mathrm{kV}$ BSE image of sintered stainless using ZT scintillator

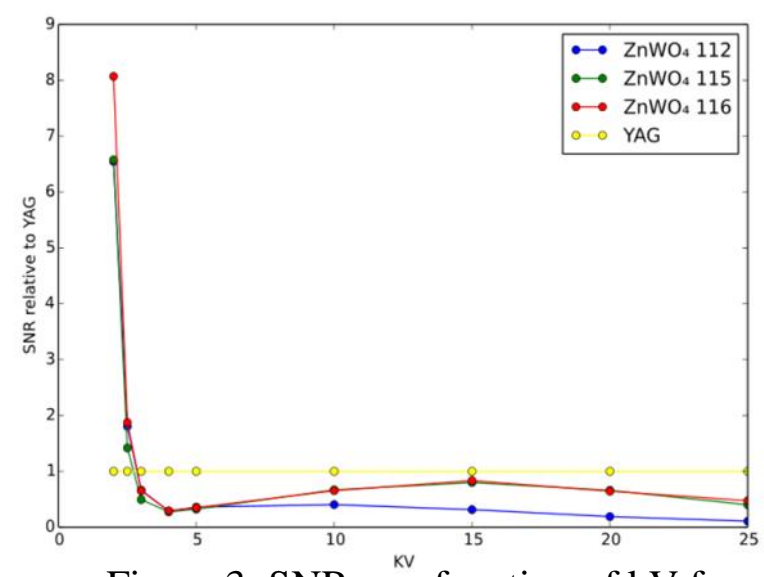

Figure 3: SNR as a function of $\mathrm{kV}$ for ZT and YAG images

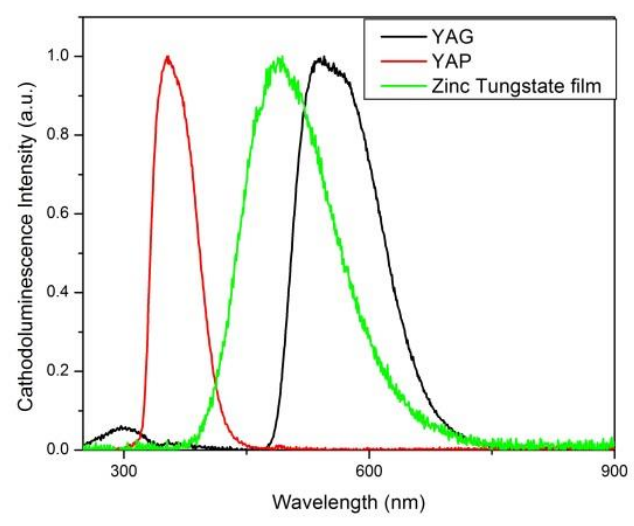

Figure 5: Emission spectra for YAG, ZT, and YAP 